\title{
La mercantilización de la producción y de la difusión del conocimiento y sus efectos. Los universitarios como sujetos sujetados
}

Fernando Acevedo

\section{RESUMEN}

Este ensayo ofrece una perspectiva teórica y política sobre la expansión de la educación superior en América Latina que difiere de la predominante en el mainstream académico actual. Con base en una investigación bibliográfica, el análisis enfatiza sus efectos más nocivos en el quehacer de los universitarios, principalmente los ocasionados por la creciente mercantilización de la producción y difusión del conocimiento que producen: sobrevaloración de la publicación de papers en revistas prestigiosas, devaluación de la utilidad social del conocimiento, prácticas corruptas, erosión de la credibilidad y de la calidad de la producción académica, competencia inescrupulosa, individualismo exacerbado, deterioro de las relaciones interpersonales.

Palabras clave: educación superior, expansión educativa, mercantilización de la educación, producción del conocimiento, publicaciones científicas, papel de la universidad. 


\section{A mercantilização da produção e da difusão do conhecimento e seus efeitos. Os universitários como sujeitos sujeitados}

\section{RESUMO}

Este ensaio oferece uma perspectiva teórica e política sobre a expansão da educação superior na América Latina que diferencia-se da predominante no mainstream acadêmico atual. Com base em uma pesquisa bibliográfica, a análise enfatiza seus efeitos mais nocivos no trabalho dos universitários, principalmente os ocasionados pela crescente mercantilização da produção e difusão do conhecimento que produzem: sobrevalorização da publicação de papers em revistas prestigiosas, desvalorização da utilidade social do conhecimento, práticas corruptas, erosão da credibilidade e da qualidade da produção acadêmica, competição inescrupulosa, individualismo exacerbado, deterioro das relações interpessoais.

Palavras chave: educação superior, expansão educativa, mercantilização da educação, produção do conhecimento, publicações científicas, papel da universidade.

The commodification of the production and dissemination of knowledge and its effects. The university students submitted subjects

\section{ABSTRACT}

This essay offers a theoretical and political perspective on the expansion of higher education in Latin America that differs from the predominant one in the current academic mainstream. Based on a bibliographic research, the analysis emphasizes its most harmful effects on the work of university students, mainly those caused by the growing commercialization of the production and dissemination of knowledge that tends to produce overvaluation of the publication of papers in prestigious journals, devaluation of the social utility of knowledge, corrupt practices, erosion of the credibility and of the quality of academic production, unscrupulous competition, exacerbated individualism and the deterioration of interpersonal relationships.

Keywords: higher education, educational expansion, commercialization of education, knowledge production, scientific publications, role of the university. 


\section{Introducción: la expansión de la educación superior en América Latina}

La dinámica y las modalidades de la expansión de la educación superior (ES) en América Latina constituyen una problemática de relevancia creciente en la producción académica regional. ${ }^{1}$ Tal como ha subrayado Ristoff (1999), en las últimas décadas la ES se ha convertido en un imperativo estratégico prioritario para la mayoría de los países de la región en virtud de la importante contribución que puede ofrecer para el desarrollo de esos países, siempre que su expansión promueva una mayor equidad y justicia social, aumentando así el carácter democrático, inclusivo, emancipador y sustentable de cada sociedad.

Los datos disponibles muestran con nitidez que en las últimas dos décadas el acceso a la ES en América Latina ha crecido en forma significativa (Cambours y Gorostiaga, 2019), aunque con ritmos y magnitudes diferentes en cada país, expresión de una marcada heterogeneidad intrarregional (Ezcurra, 2019). En cualquier caso, el incremento sostenido de la matrícula parece indicar que la masificación en la ES ya es una tendencia global e irreversible (Ezcurra, 2011a), pero por sí mismo nada dice sobre los beneficios que eventualmente pueda implicar, es decir, si se trata de un incremento deseable per se, o con consecuencias beneficiosas per se. En efecto, "en la expansión educativa, la cuestión clave es si reduce la desigualdad al proveer más oportunidades a personas de estratos sociales en desventaja, o si la magnifica al ampliar de modo desproporcionado las oportunidades de aquellos que ya son privilegiados" (Arum et al., 2007: 124).

En este sentido, son de especial interés las diversas hipótesis sobre los efectos de la masificación en la igualdad social, o bien en la desigualdad social, formuladas por Ezcurra (2011b): ingreso a la ES de sectores sociales que han permanecido excluidos - masificación inclusiva-, inclusión estratificada según posición social -inclusión y desigualdad-, desigualdad diversa en el ingreso, en la permanencia y en la graduación, y aparición de nuevas formas de desigualdad social, entre ellas una inclusión centrífuga o excluyente.

Pero es necesario, también, mirar al sesgo.

\section{El desafío a enfrentar en América Latina en un contexto de expansión del acceso a la educación superior}

Mirar al sesgo los temas teóricos no es sólo un intento orientado a hacer más accesible la teoría, y entonces ahorrarnos el trabajo de pensar; responde, más bien, a la convicción de que "esa escenificación de los temas teóricos saca a la luz ciertos aspectos que de otro modo seguirían inadvertidos" (Žižek, 2002: 17). Además de delinear el perfil general del edificio teórico sobre la expansión de la ES en algunos países de América Latina, mirar al sesgo también permite dar cuenta de "los detalles menudos que la recepción predominantemente académica [...] suele pasar por alto [y] discernir rasgos que por lo general se sustraen a una mirada académica "de frente"' (ibid.: 10).

Un "detalle" en absoluto menudo es que el fenómeno de la expansión de la ES no se agota en la cuestión de la magnitud y de las modalidades y efectos del incremento del acceso a ese nivel, de enorme e inequívoca relevancia. Muchos especialistas en la materia (por ejemplo, Arocena y Sutz, 2016; Cambours de Donini y Gorostiaga, 2016; Ristoff, 2011; Silva Júnior y Catani, 2013) coinciden en destacar la importancia que reviste la ES - y su expansión - para enfrentar, en un marco de equidad social, las exigencias y desafíos crecientes del mundo contemporáneo.

\footnotetext{
${ }^{1}$ El contenido del desarrollo argumental del presente ensayo se nutrió de la producción bibliográfica sobre la temática abordada y de los planteos expuestos en el $2^{\circ}$ Foro Educativo Regional organizado por el Centro de Estudios sobre Políticas Educativas de la Universidad de la República, Uruguay. El Foro, cuyo tema central fue "La expansión de la educación superior en América Latina", tuvo lugar en la fronteriza ciudad de Rivera, Uruguay, el 4 de septiembre de 2019. Los expositores centrales en el Foro fueron cuatro renombrados académicos de la región: Afrânio Catani (Universidad de São Paulo, Brasil), Jorge Gorostiaga (Universidad Nacional de San Martín, Argentina), Gregory Randall (Universidad de la República, Uruguay) y Dilvo Ristoff(Universidad de Santa Catarina, Brasil).
} 
Siendo así, además del aumento del acceso a la ES en procura de una mayor equidad social y de la provisión de una enseñanza de calidad para todos, también resulta crucial la expansión y optimización de la investigación y de la extensión universitaria, que son otras dos funciones tradicionales inherentes a la ES y en especial a la universidad, en tanto institución social centrada en la producción y la difusión de conocimiento; se trata de dos funciones que, naturalmente, también deben cumplir a cabalidad con los requisitos de calidad, equidad y justicia social.

El desarrollo del capitalismo global, monopolista y de predominio financiero, en gran parte del mundo ha dado lugar a la instalación de un proceso de mercantilización de la producción y de la difusión del conocimiento que se produce en las universidades (Burawoy, 2015). Entre sus principales efectos se destaca el gran crecimiento de las universidades privadas como brazos del capital corporativo, en detrimento de las públicas, la proliferación de rankings mundiales, regionales y nacionales, la profundización del rol de las universidades como máquinas expendedoras de credenciales (Calderón et al., 201 1), la consolidación de la concentración de recursos en las universidades más ricas y su dominación del campo al momento de establecer los problemas a investigar y las modalidades más adecuadas para su abordaje.

En este contexto, el gran desafío a enfrentar, como ha destacado Catani (2009) para el caso de Brasil, es expandir la ES pública, gratuita y con estándares de calidad aceptables, de acuerdo con parámetros aceptados universalmente, en instituciones en las que enseñanza, investigación y extensión caminen juntas, integradas. En Argentina y en Uruguay la primera parte del desafío se está cumpliendo de modo satisfactorio; empero, por lo menos en las organizaciones de ES de Uruguay, aún no se ha logrado que enseñanza, investigación y extensión caminen efectivamente integradas. Esbocemos alguna explicación plausible.

\section{Principales efectos nocivos de la mercantilización de la producción y de la difusión del conocimiento}

El proceso globalizado de mercantilización de la producción y de la difusión del conocimiento ha conducido a que la investigación, tanto dentro como fuera de las universidades, quede subsumida en la lógica de la mundialización del capital, de su producción y circulación. En los ámbitos editoriales inscritos en esa lógica (journals, revistas especializadas de alto impacto internacional, en su gran mayoría privadas y anglófonas), que son aquellos en los que los investigadores universitarios se ven cada vez más impelidos a publicar sus obras, la utilidad social del conocimiento producido en las universidades, sobre todo en el campo de las ciencias sociales y humanas, está lamentablemente devaluada.

Tal como se expresa en el Manifiesto de Leiden, en muchas partes del mundo excelencia en investigación se asocia únicamente con publicaciones en inglés (Hicks et al., 2015). En muchos países la normativa explicita la conveniencia de que los investigadores publiquen en revistas de alto impacto, ${ }^{2}$ pero el factor de impacto se calcula exclusivamente para revistas indexadas por Web of Science, que en su enorme mayoría son en inglés. Este sesgo es bastante más problemático en las ciencias sociales y humanas, campos en los que la investigación suele estar más orientada a temas regionales y locales y en los que las inequidades inherentes a la geopolítica del conocimiento son más manifiestas e inhiben la consolidación de "un pensamiento propio [y crítico] latinoamericano [...] como reacción frente a modelos académicos que se

\footnotetext{
${ }^{2}$ En los países hispanohablantes el caso más notorio es el de España; lo mismo ocurre en Uruguay, aunque de modo menos explícito, en las instancias de evaluación de los investigadores, tanto por parte del Sistema Nacional de Investigadores (un programa gubernamental de incentivos económicos a investigadores) como de la Universidad de la República, la institución que conjuga la mayor tradición, volumen poblacional y relevancia social en el país.
} 
consideran intrusivos" (Mignolo, 2001: 20). En cualquier caso, algunos de los efectos de esas inequidades se evidencian en la muy baja cantidad de universidades latinoamericanas - apenas diez ${ }^{3}{ }^{3}$ incluidas en los principales rankings mundiales y en la posición muy marginal que ocupan en ellos (Maldonado, 2017).

A la devaluación de la utilidad social del conocimiento producido en las universidades también contribuyó la intensidad con la que el individualismo neoliberal opera en este campo (Borón, 2020) y su efecto en la degradación de lo social: lo social se alienta sólo si contribuye a optimizar las relaciones de producción (Han, 2014a).

Por lo pronto, hace tiempo que la utilidad social ha dejado de ser un criterio de calidad para la evaluación de la producción académica, si es que alguna vez lo fue en un grado apreciable. La ideología neoliberal imperante, subsidiaria de la lógica del capital, deja a un lado la utilidad social del conocimiento y restringe el espacio de la cooperación interpersonal, al tiempo que privilegia los aspectos rentables y exacerba la competencia.

En este contexto, a las presiones del sistema sobre el productor académico se suma, con mayor fuerza, su propia auto-exigencia: "Quien fracasa en la sociedad neoliberal del rendimiento se hace a sí mismo responsable y se avergüenza, en lugar de poner en duda a la sociedad o al sistema. En esto consiste la especial inteligencia del régimen neoliberal" (Han, 2014b: 18); éste opera, naturalmente, favoreciendo la eficacia del sistema: "La propia explotación es más eficaz que la explotación extraña, pues va acompañada del sentimiento de libertad. El sujeto del rendimiento se somete a una coacción libre, generada por él mismo" (Han, 2013: 92). Esta auto-explotación - el sujeto no dirige su resistencia contra el sistema sino contra sí mismo - pone en evidencia el influjo de la astucia del régimen neoliberal (Han, 2014b).

En esta lógica de mercantilización, las revistas científicas - en tanto son, en su enorme mayoría, privadas - alientan la privatización de un bien de carácter público; el conocimiento se convierte en un commodity que los imperios editoriales oligopólicos negocian por volumen más que por la calidad de su contenido. ${ }^{4}$ Se instala, así, un feroz escenario hobbesiano en el que los investigadores ponen todo de sí para que los journals les publiquen sus papers - $\mathrm{y}$ para que sus colegas los citen, cuantos más mejory las universidades para alcanzar puestos altos en los rankings globales (por ejemplo, en el listado del Times Higher Education o en el ranking de Shanghai), ya que a escala internacional la cantidad de papers publicados es uno de los principales indicadores para evaluar a las universidades. Pero el empleo de métricas cuantitativas para la elaboración de rankings o para la evaluación del impacto de papers y journals, ${ }^{5}$ aun cuando esté bien intencionado, puede dar lugar a inequidades y producir resultados peores que los sistemas que reemplazaron (Edwards y Roy, 2017).

\footnotetext{
${ }_{3}^{3}$ Las universidades aludidas por Maldonado (2017) son las siguientes: Universidade de São Paulo, Universidad Nacional Autónoma de México, Universidade Estadual de Campinas, Universidade Federal do Rio de Janeiro, Universidade Estadual Paulista, Universidade Federal de Minas Gerais, Pontificia Universidad Católica de Chile, Universidad de Chile, Universidad de Buenos Aires y Universidad de Los Andes (Colombia).

${ }^{4} \mathrm{Si}$ consideramos que en la actualidad se publican unos tres millones de artículos científicos por año (Lorite, 2019), es indudable que muchas revistas académicas son hoy un negocio suculento: por ejemplo, el mayor conglomerado editorial actual "publica alrededor de 2500 revistas académicas, tiene unos 16 millones de artículos en sus repositorios y, según datos de 2018, tiene una ganancia de $37 \%$ de su facturación" (Lagos, 2019), unos 22 mil millones de euros, muy superior a las ganancias de empresas como Google, Apple o Amazon (Martínez Ron, 2017). Algo similar ocurre con el resto de las editoriales que oligopolizan el mercado de publicaciones científicas Cambridge Scholar Press, Springer Nature, Wiley-Blackwell, De Gruyter, Taylor \& Francis - , que tienen, a costa del trabajo de los investigadores, márgenes de ganancia de hasta el 40\% (Berger et al., 2018). Quienes subsidian a esas empresas y propician tan alta rentabilidad no son sólo las universidades - que son las que les pagan a los investigadores-y los propios investigadores, que trabajan gratis como revisores, pagan por leer los papers de sus colegas y regalan sus propios artículos —o, en revistas de acceso abierto, los venden-; también lo hace el resto de los ciudadanos, al contribuir con sus impuestos al sustento económico de las universidades.

${ }^{5} \mathrm{El}$ prestigio de una revista es directamente proporcional a su factor de impacto (fournal Impact Factor, JIF), un índice bibliométrico que
} 
Esta suerte de "paperfilia" ya está sólidamente instalada y, en tanto expresión del proceso de mercantilización inherente al capitalismo (Han, 2014b), parece ser un fenómeno irreversible. En este contexto, los papers publicados, los factores de impacto de las revistas y los rankings se erigen como fines en sí mismos, casi con carácter de fetiches. Bastante antes de que se consolidara este escenario, según una sentencia atribuida a Einstein, algo distintivo de la ciencia era (en su época) la perfección de medios y la confusión de fines; una derivación previsible está recogida en la denominada Ley de Goodhart, reivindicada por Edwards y Roy (2017): cuando una medición se vuelve un fin, deja de ser una buena medición. La perfección de medios y la confusión de fines sigue siendo una combinación vigente, aunque los medios actuales - rankings, papers, factores de impacto, métricas e indicadores cuantitativos de productividad, etcétera - distan mucho de ser perfectos. ${ }^{6}$ Una notoria "imperfección" es que las mediciones no consideran los contextos socio-culturales, económicos y académicos en los que se producen las investigaciones ni distinguen si se trata de investigación básica o aplicada ni los campos disciplinares en los que se sustentan teórica y metodológicamente, ya que las prácticas de producción, de publicación y de citación en un campo científico difieren con las de otros. Además de que distan mucho de ser perfectos, esos medios producen efectos que pueden llegar a ser perversos.

Los efectos más nocivos conducen a la erosión de la credibilidad y de la calidad de la producción científica que se publica y, por extensión, de la producción científica en general. Freeland (2004), por ejemplo, destaca el aumento de malas prácticas y de falseamiento de resultados en los papers, lo cual, para Smaldino y McElreath (2016), conduce a una selección natural de mala ciencia. En un sentido concordante Edwards y Roy (2017) afirman que los factores y las métricas pueden ser manipuladas - y que a menudo lo son- y que la presión que las instituciones ejercen sobre los académicos los lleva a privilegiar la cantidad de papers publicados en detrimento de su calidad: los papers son cada vez más breves, superficiales y pobres metodológicamente. Asimismo, como apunta Lorite (2019), se multiplican los refritos, esto es, la reformulación de papers ya publicados a efectos de que sus autores puedan mantener el ritmo exigido de publicación.

Edwards y Roy (2017) aseveran que la calidad de las revisiones (peer reviezes) se ha reducido, a lo que se añaden preocupantes conductas inmorales, como por ejemplo la solicitud a los autores, por parte de algunos revisores, de que citen sus propios artículos para que sus papers sean aceptados. Otra de las prácticas, mencionada por Lorite (2019), es que grupos de investigadores académicamente bien posicionados envían unos cuantos papers a alguna revista de prestigio relativamente bajo y se citan entre sí para aumentar su posicionamiento (y, por ende, el de la revista).

En definitiva, una considerable cantidad de investigadores, enajenados por las métricas y a contramano de sus auténticos intereses de investigación, con el propósito de que sus papers sean publicados en revistas de alto impacto internacional incurren en conductas claramente reñidas con la ética profesional.

es el resultado del cociente entre el número de citas que recibe una revista y el número de artículos que publica. ElJIF también se aplica como "indicador cuantitativo de la valía de los sujetos productores de ciencia, bajo la premisa de que dicha valía viene dada por el prestigio de las revistas donde publican” (Soriano, 2018). Según Edwards y Roy (2017), desde la Segunda Guerra Mundial el JIF se ha duplicado cada nueve años.

${ }^{6}$ Por ejemplo, los factores de impacto de las revistas más citadas en los rankings de biología celular son diez veces mayores que los de las revistas más citadas en los rankings de matemática (Hicks et al., 2015). En cualquier caso, un cómputo alto de citas no necesariamente da cuenta cabal de la calidad de un paper (ni de la excelencia de un journal); solamente expresa que un fragmento de ese paper ha sido usado, pero nada dice sobre el carácter de ese uso - si es elogioso o reprobatorio - ni si se trata de una cita de una cita. En efecto, la cuantificación de las citas que recibe un artículo no permite inferir nada con respecto a su calidad ni a la de los autores de los artículos ni a la de las instituciones en las que se desempeñan. Esto también vale para cualquier índice bibliométrico basado en las citas (Soriano, 2018). 
(También ocurre que algunos directores de proyectos instan a "sus" investigadores a que en sus papers incorporen como coautores a colegas que no participaron en la investigación.) La conclusión de Edwards y Roy (2017), reafirmada por Meagan Day (2018), es lapidaria: si nada se hace al respecto, habremos de crear una cultura académica corrupta.

\section{La efectiva integración de enseñanza, investigación y extensión, una aspiración cada vez más esquiva}

Los principales efectos de la mercantilización de la producción y de la difusión del conocimiento reseñados en el apartado precedente - la privatización y la devaluación de la utilidad social del conocimiento producido en las universidades, la tendencia cuantofrénica, la erosión de la credibilidad y de la calidad de la producción científica, la competencia inescrupulosa, el individualismo exacerbado, la emergencia de otros aspectos miserables del yo, los papers como fetiches, el deslizamiento hacia prácticas académicas corruptas - contribuyen a obturar el logro de una efectiva integración de enseñanza, investigación y extensión.

Lo que aquí interesa destacar es que esos efectos, y en especial la hipervaloración de la publicación de papers y de los factores de impacto como fines en sí mismos, dan lugar a una situación que se constituye como un difícil obstáculo para aquel logro: muchos docentes universitarios deciden privilegiar sus actividades de investigación - siempre teniendo en la mira la escritura de papers a ser enviados a algunos journals de presunto alto impacto internacional- en desmedro de las actividades de enseñanza y de extensión universitaria.

Si obviamos el perjuicio que esta decisión le ocasiona a la mayoría de los destinatarios de la enseñanza - los estudiantes - y de la extensión universitaria - ciudadanos que gracias a ella podrían mejorar su calidad de vida-, quedamos en condiciones de admitir que se trata de una decisión entendible, ya que es sensato que en todo ámbito laboral el trabajador priorice la realización de aquellas tareas a las que se somete la evaluación de su desempeño y, entonces, pueda asegurar su permanencia en su cargo.

En el ámbito universitario latinoamericano actual la publicación de papers en revistas de alto impacto internacional es el item de mayor valor en los baremos que se aplican en la evaluación del desempeño profesional del docente (por ejemplo, al momento de la renovación de su contrato o de su postulación a un cargo académico de mayor jerarquía), ${ }^{7}$ mientras que la evaluación de sus actividades de enseñanza y de extensión universitaria suele ser, cuando existe, accesoria y bastante laxa. En consecuencia, es muy frecuente que los profesores universitarios más calificados ocupen la mayor parte de su tiempo en investigar para publicar, reduciendo el tiempo que le destinan al dictado de cursos y, sobre todo, a la preparación de clases, atención a estudiantes, escritura de libros de divulgación, ${ }^{8}$ actividades de

\footnotetext{
${ }^{7}$ En la evaluación lo que más cuenta es la cantidad de papers publicados y no su calidad. La calidad cuenta, sí, pero "filtrada" con cierta dosis de discrecionalidad, ya que los evaluadores la infieren de la posición que ocupa la revista en los rankings (por ejemplo, en los que aplica Web of Science, si se trata de la categoría Q1, Q2, Q3 o Q4). Este tipo de evaluación resulta muy cómoda para los evaluadores, ya que no necesitan ocupar su tiempo en leer los artículos completos para determinar su calidad: cuanto mejor calificada está la revista — cuanto mayor es su JIF- más puntaje se les atribuye a los artículos publicados en ella (Hernández, 2015).

${ }^{8} \mathrm{En}$ las instancias de evaluación los libros publicados obtienen un puntaje muy inferior a los papers indexados en las principales bases de datos (por ejemplo, Web of Science y Scopus). Esto no solamente ocurre en nuestra región. En España, por ejemplo, las evaluaciones de la Agencia Nacional de Evaluación de la Calidad y Acreditación (ANECA) adjudican 0 puntos a los libros de divulgación. Por razones que por limitación de espacio no se expondrán aquí, esto suele perjudicar bastante más a los investigadores del campo de las ciencias sociales y humanas. Basta con señalar que en este campo por lo general es mucho mayor el impacto social de los libros que el de los papers, entre otras cosas porque a éstos accede una élite de muy pocas personas, la mayoría de las cuales solamente lee los abstracts (ya sea porque no disponen de tiempo para leer el paper completo o porque deben pagar para hacerlo). Debe advertirse, además, que la disminución de la publicación de libros de divulgación contribuye a que muchos ciudadanos condenen lo que perciben como aislamiento social de la universidad y a los docentes universitarios como atrincherados en torres de marfil.
} 
relacionamiento y de intercambio de conocimientos con el medio social, etcétera.

De hecho, por lo menos en el caso de Uruguay, los docentes universitarios que le dedican la mayor parte de su carga horaria a actividades de extensión - función enfáticamente destacada en los discursos y documentos oficiales universitarios - suelen tener grados académicos más bajos que los que se dedican en forma mayoritaria a actividades de enseñanza - función sine qua non de la universidad-, y éstos grados académicos más bajos que los que le dedican la mayor parte de su jornada laboral a la investigación... y, sobre todo, a recorrer el febril proceso orientado a la publicación de papers. ${ }^{9}$

Además, no se debe soslayar que las herramientas con las que cuentan los agentes universitarios son, en su mayoría, cada vez menos adecuadas para valorar a aquellos docentes que, pudiendo hacerlo, no se involucran en la competencia por publicar papers y optan, en su lugar, por privilegiar actividades de enseñanza y de extensión universitaria, de enorme importancia social y política.

\section{Consideraciones finales: los universitarios como sujetos sujetados}

La expansión de la educación superior pública en América Latina es un proceso ya consolidado y, seguramente, irreversible. El acceso a ese nivel de sectores sociales antes excluidos y las acciones que se ejecutan para promover la persistencia y el éxito de los estudiantes ${ }^{10}$ tienen un efecto democratizador que debe ser enfáticamente destacado. En esa línea se inscribe el ya citado planteo de Catani (2009): el gran desafio a enfrentar es expandir la educación superior pública, gratuita y con estándares aceptables de calidad, en instituciones en las que enseñanza, investigación y extensión caminen juntas, integradas.

No obstante, la expansión de la ES pública no debe restringirse a la ampliación y democratización del acceso. De acuerdo con lo expuesto antes, la creciente primacía de la lógica del capital y de la competencia inherente a la ideología neoliberal dificulta en alto grado la posibilidad de alcanzar estándares elevados de calidad y, sobre todo, de que las funciones de enseñanza, investigación y extensión se desempeñen de forma genuinamente integrada.

Más relevante aun, la sujeción a la lógica del capital está alejando a la ES pública de su sentido profundo, viciando la convivencia entre actores universitarios y, en cierta medida, erosionando algunos atributos sustantivos que las universidades comenzaron a forjar desde la irrupción del movimiento de Córdoba, hace ya cien años: la autonomía de pensamiento, la reflexión crítica, la deliberación sistemática, la cooperación interpersonal, todo ello orientado a la transformación social con base en la solidaridad y en la justicia social como tarea colectiva. Siendo así, tal vez debamos avalar la percepción de Žižek (2002: 12) en cuanto a "la inconmensurabilidad fundamental que existe entre el dominio simbólico de la igualdad, los derechos, los deberes, etcétera, y la particularidad absoluta del espacio fantasmático, es decir, de los modos específicos en que los individuos y las comunidades organizan su goce".

Hecho eso, se vuelve turbador reconocer que una cantidad creciente de universitarios, orgullosos y autoproclamados partícipes de comunidades académicas, y sometidos a potentes exigencias institucionales e imperativos externos, organizan su goce - cuando no su miedo - en torno a la pulsión de encontrar sus nombres estampados encima de los abstracts de cada paper que febrilmente publican.

\footnotetext{
${ }^{9}$ Cualquiera que frecuente espacios comunes de centros universitarios (cafeterías, atrios, corredores) podrá observar que en las conversaciones informales entre docentes de alto grado académico — también entre los que aspiran firmemente a alcanzar ese estatuspredomina tácitamente cierta visión que descalifica a los "meros enseñantes", más descarnada aun con respecto a los docentes "extensionistas".

${ }^{10}$ Aquí entendemos al éxito de los estudiantes en el sentido atribuido por Tinto (2012) a la expresión "student success".
} 
Pero no se trata, en absoluto, de culpabilizar a las víctimas: los actores universitarios somos, mal que nos pese, sujetos sujetados. Si bien muchos académicos nos han mostrado la lógica y algunos mecanismos de la sujeción, hasta el momento no hemos sido capaces de mitigarla, y mucho menos de resistirla.

Uno de los caminos a nuestro alcance pasa por capitalizar el impulso y las potencialidades de las nuevas generaciones, habida cuenta de que a los que recién se incorporan al mundo académico - estudiantes, docentes noveles - tal vez les resulte más dificil reconocer que se los acuesta en un cruel lecho de Procusto, y que no llegará ningún hermano, ningún Teseo, a hacer justicia. ${ }^{11}$

$\mathrm{Al}$ igual que en tantos otros ámbitos sociales, contribuir a forjar ese reconocimiento es tarea de todos. En definitiva, es cada vez más imperioso asumir la máxima que la coalición Herri Batasuna tomara de Jesús Ibáñez (1994): cuando algo es necesario e imposible, hay que cambiar las reglas del juego con el que juegan con nosotros.

\footnotetext{
${ }^{11}$ Dice un mito griego que Procoptas, hijo del dios de los mares, ofrecía alojamiento a viajeros ocasionales en su modesta vivienda cerca de la ciudad sagrada de Eleusis. Allí, luego de rapiñarlos, los obligaba a acostarse desnudos en un catre de hierro. A quienes les quedaba chico Procoptas los mutilaba con un hacha, de modo que nada sobrara en la superficie del lecho; si, en cambio, el huésped era de poca talla, con ayuda de tiras de cuero atadas a la cabecera y a los pies del catre le estiraba las extremidades hasta que cubrieran los sectores vacíos. Esas prácticas le valieron unos cuantos alias: Damastes (el abusador), Polipemón (el dañino) y Procrustes (el estirador); este último, el más difundido, acabó transformándose en Procusto. Ciertamente, las disposiciones y dispositivos impuestos a menudo funcionan como el lecho de Procusto: se acuesta sobre él a las víctimas, sin que importen las características singulares de cada una de ellas, y se las acomoda, cueste lo que cueste, a las dimensiones de aquél. Muchos yacerán indemnes, pero unos cuantos quedarán descoyuntados, tullidos, mancos o cojos. En cualquier caso, es doloroso y humillante tener que sufrir tal tormento. Afortunadamente, hubo quien recicló el lecho de Procusto. Teseo, también hijo de Poseidón, el mismo que tiempo antes, con la ayuda de Ariadna, había matado al Minotauro, atrapó a su hermano Procusto y — con similar inspiración que la de sus coetáneos administradores de justicia, desde el código de Hammurabi hasta la ley de Talión instaurada en el Levítico - lo sometió a la tortura del catre que el propio Procrustes, el estirador, gustaba de aplicar a sus víctimas.
} 


\section{Referencias}

Arocena, Rodrigo y Judith Sutz (2016), Universidades para el desarrollo, Montevideo, UNESCO.

Arum, Richard, Adam Gamoran y Yossi Shavit (2007), "More inclusion than diversion: expansion, differentiation, and market structure in higher education", en Yossi Shavit, Richard Arum y Adam Gamoran (eds.), Stratification in Higher Education. A comparative study, Stanford, Stanford University Press, pp. 1-35.

Berger, Juan José, María Loreto y Antonella Rescaglio (2018), Qué hacer para cambiar las reglas que someten la investigación científica al mercado del conocimiento, <https:/ / ciperchile.cl/2018/12/03/que-hacer-para-cambiarlas-reglas-que-someten-la-investigacion-cientificaal-mercado-del-conocimiento/> [Consulta: julio de 2019].

Borón, Atilio (2020), "La crisis de las ciencias sociales y el papel de la universidad", Granma, La Habana, 21 de febrero.

Burawoy, Michael (2015), "Ensino superior em crise: o contexto global", Margem Esquerda: ensaios marxistas, núm. 25, pp. 43-51.

Calderón, Adolfo, Rodrigo Fornalski y María Caroline Vargas (2011), "Responsabilidade social da educação superior: a metamorfose do discurso da UNESCO em foco", Interface-Comunicação, Saúde, Educação, núm. 15, pp. 85-98.

Cambours de Donini, Ana María y Jorge Gorostiaga (2019), "Acceso y permanencia en universidades del Conurbano: logros y límites de las políticas institucionales", en Ana María Ezcurra (coord.), Derecho a la educación. Expansión y desigualdad: tendencias y políticas en Argentina y América Latina, Sáenz Peña, EDUNTREF.

Cambours de Donini, Ana María y Jorge Gorostiaga (coords.) (2016), Hacia una universidad inclusiva. Nuevos escenarios y miradas, Buenos Aires, Aique Grupo Editor.

Catani, Afrânio (2009), "Expansão do acesso à educação superior no Brasil: alguns desafios", Tempo Brasileiro, núm. 178, pp. 111-123.
Day, Meagan (2018), "Capitalism is ruining science", facobin, 9 de julio, <https://www.jacobinmag. com/2018/07/capitalism-science-research-academiafunding-publishing > [Consulta: enero de 2020].

Edwards, Marc y Siddhartha Roy (2017), "Academic research in the 21st Century: maintaining scientific integrity in a climate of perverse incentives and hypercompetition", Environmental Engineering Science, núm. 34 (1), <https://www.liebertpub.com/doi/10.1089/ ees.2016.0223> [Consulta: enero de 2020].

Ezcurra, Ana María (2019), "Educación superior: una masificación que incluye y desiguala”, en Ana María Ezcurra (coord.), Derecho a la educación. Expansión y desigualdad: tendencias y políticas en Argentina y América Latina, Sáenz Peña, EDUNTREF.

Ezcurra, Ana María (2011a), Igualdad en educación superior. Un desafio mundial, Buenos Aires, Editorial Universidad Nacional de General Sarmiento.

Ezcurra, Ana María (2011b), "Masificación y enseñanza superior. Algunas hipótesis y conceptos clave", en Norberto Fernández Lamarra y María Costa de Paula (eds.), La democratización de la educación superior en América Latina: limites y posibilidades, Buenos Aires, EDUNTREF.

Freeland, Horace (2004), The great betrayal: fraud in science, Orlando, Harcourt.

Han, Byung-Chul (2014a), En el enjambre, Barcelona, Herder.

Han, Byung-Chul (2014b), Psicopolitica. Neoliberalismo y nuevas técnicas de poder, Barcelona, Herder.

Han, Byung-Chul (2013), La sociedad de la transparencia, Barcelona, Herder.

Hernández, Esteban (2015), La "cuantofrenia": una definición de la estupidez que impera en la universidad, <https://www. elconfidencial.com/alma-corazon-vida/2015-04-08/lacuantofrenia-una-definicion-de-la-estupidez-que-imperaen-la-universidad_749102/> [Consulta: julio de 2019].

Hicks, Diana, Paul Wouters, Ludo Waltman, Sarah de Rijcke e Ismael Rafols (2015), "The Leiden Manifesto for research metrics”, Nature, núm. 520, pp. 429-431. 
Ibáñez, Jesús (1994), El regreso del sujeto. La investigación social de segundo orden, Madrid, Siglo XXI.

Lagos, Leo (2019), “¿Dónde y cómo publicarán nuestros investigadores sus artículos científicos?", $L a$ Diaria, 27 de agosto, <https://ciencia.ladiaria.com.uy/ articulo/2019/8/donde-y-como-publicaran-nuestrosinvestigadores-sus-articulos-cientificos $/>\quad$ [Consulta: agosto de 2019].

Lorite, Álvaro (2019), "Papers" y más "papers": las sombras en la industria de las publicaciones cientificas, <https://www. elsaltodiario.com/universidad/papers-y-mas-paperslas-sombras-en-la-industria-de-las-publicacionescientificas?> [Consulta: enero de 2020].

Maldonado, Alma (2017), "La inequidad en la producción y el uso del conocimiento ¿dónde queda América Latina?", en Laura Phillips (comp.), Construcción de espacios regionales: inclusión social latinoamericana, Bogotá, Universidad de Ciencias Aplicadas y Ambientales/ Asociación de Universidades de América Latina y el Caribe para la Integración, pp. 57-69, <http://www.die. cinvestav.mx/Portals/die/SiteDocs/Investigadores/ AMaldonado/CapLibros/AMM_Inequidad \%20 AL\%20Capt..pdf?ver=2018-06-06-1 12738-100> [Consulta: julio de 2020].

Martínez Ron, Antonio (2017), “¿Está rota la máquina de hacer ciencia?", Revista digital Next. Ciencia y Futuro, <https://www.vozpopuli.com/altavoz/next/rotamaquina-hacer-ciencia_0_1047795794.html> [Consulta: diciembre de 2019].
Mignolo, Walter (2001), "Introducción", en W. Mignolo (comp.), Capitalismo y geopolítica del conocimiento: el eurocentrismo y la filosofia de la liberación en el debate intelectual contemporáneo, Buenos Aires, Ediciones del Signo, pp. 9-53.

Ristoff, Dilvo (1999), Universidade em Foco. Reflexões sobre a Educação Superior, Florianópolis, Insular.

Silva Júnior, João dos Reis y Afrânio Catani (2013), "A educação superior pública brasileira nas duas últimas décadas: expansão e mercantilização internacionalizada", en José Vieira de Sousa (org.), Educação superior: cenários, impasses e propostas, Campinas, Autores Associados, pp. 157-181.

Smaldino, Paul y Richard McElreath (2016), The natural selection of bad science, <https://royalsocietypublishing. org/doi/full/10.1098/rsos.160384> [Consulta: octubre de 2019].

Soriano, Carles (2018), Ciencia, publicaciones cientificas y cienciometría. Una aproximación crítica, <http.//www. bitacora.com.uy/auc.aspx?7050,7> [Consulta: octubre de 2019].

Tinto, Vincent (2012), "Moving from Theory to Action. A Model of Institutional Action for Student Success", en Alan Seidman (ed.), College student retention. Formula for student success, Plymouth, Rowman \& Littlefield, pp. 251-266.

Žižek, Slavoj (2002) [1991], Mirando al sesgo. Una introducción a Jacques Lacan a través de la cultura popular, Buenos Aires, Paidós.

\section{Cómo citar este artículo:}

Acevedo, Fernando (2021), "La mercantilización de la producción y de la difusión del conocimiento y sus efectos. Los universitarios como sujetos sujetados", Revista Iberoamericana de Educación Superior (RIES), vol. XII, núm. 34, pp. 145-155, DoI: https://doi. org/10.22201/iisue.20072872e.2021.34.984 [Consulta: fecha de última consulta]. 